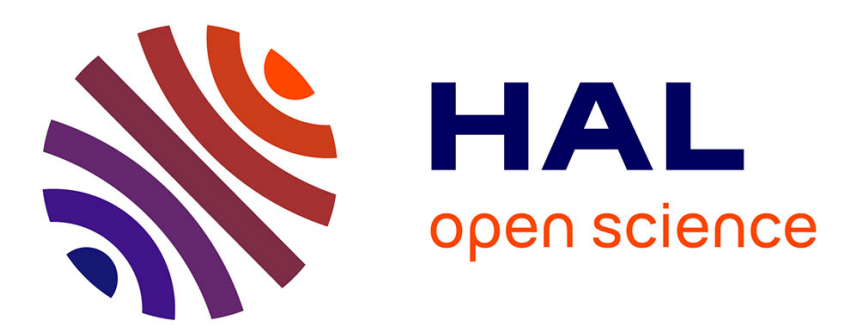

\title{
Laplacian-Regularized MAP-MRI: Improving Axonal Caliber Estimation
}

Rutger H.J. Fick, Demian Wassermann, Gonzalo Sanguinetti, Rachid Deriche

\section{To cite this version:}

Rutger H.J. Fick, Demian Wassermann, Gonzalo Sanguinetti, Rachid Deriche. Laplacian-Regularized MAP-MRI: Improving Axonal Caliber Estimation. International Symposium on BIOMEDICAL IMAGING: From Nano to Macro, Apr 2015, Brooklyn, New York City, United States. hal-01140021

\section{HAL Id: hal-01140021 \\ https://hal.inria.fr/hal-01140021}

Submitted on 7 Apr 2015

HAL is a multi-disciplinary open access archive for the deposit and dissemination of scientific research documents, whether they are published or not. The documents may come from teaching and research institutions in France or abroad, or from public or private research centers.
L'archive ouverte pluridisciplinaire HAL, est destinée au dépôt et à la diffusion de documents scientifiques de niveau recherche, publiés ou non, émanant des établissements d'enseignement et de recherche français ou étrangers, des laboratoires publics ou privés. 


\title{
Laplacian-Regularized MAP-MRI: Improving Axonal Caliber Estimation
}

\author{
R.H.J. Fick \\ D. Wassermann ${ }^{\star}$ \\ G. Sanguinetti $i^{\dagger}$ \\ R. Deriche \\ * Athena Project-Team, Inria Sophia Antipolis - Méditerranée, France \\ ${ }^{\dagger}$ Centre for Analysis, Scientific computing and Applications, TU/e - Eindhoven, The Netherlands
}

\begin{abstract}
In diffusion MRI, the accurate description of the entire diffusion signal from sparse measurements is essential to enable the recovery of microstructural information of the white matter. The recent Mean Apparent Propagator (MAP)-MRI basis is especially well suited for this task, but the basis fitting becomes unreliable in the presence of noise. As a solution we propose a fast and robust analytic Laplacian regularization for MAP-MRI. Using both synthetic diffusion data and human data from the Human Connectome Project we show that (1) MAP-MRI has more accurate microstructure recovery compared to classical techniques, (2) regularized MAP-MRI has lower signal fitting errors compared to the unregularized approach and a positivity constraint on the EAP and (3) that our regularization improves axon radius recovery on human data.
\end{abstract}

Index Terms - Diffusion MRI, MAP-MRI, Laplacian Regularization, Corpus Callosum, Axon Radius Recovery

\section{INTRODUCTION}

In diffusion MRI (dMRI), the reconstruction of the diffusion signal in q-space allows for the estimation of the water displacement probability, known as the Ensemble Average Propagator (EAP) [1]. This EAP describes the probability density that a particle will move along a certain direction in a given diffusion time $\Delta$. The EAP, or $P(\mathbf{r})$, is related to the diffusion signal by a Fourier transform $P(\mathbf{r})=\int_{\mathbb{R}^{3}} E(\mathbf{q}) e^{-2 i \pi \mathbf{q} \cdot \mathbf{r}} d \mathbf{q}$. Here $\mathbf{r}$ is a real displacement vector in $\mathbf{r}$-space and $E(\mathbf{q})$ is the normalized diffusion signal where wave vector $\mathbf{q}=$ $(\gamma \delta \mathbf{G}) / 2 \pi$ related to the applied magnetic field gradient magnitude, direction and duration [1]. The clinically used $\mathbf{b}$-value is related to $\mathbf{q}$ as $\mathbf{b}=4 \pi^{2} \mathbf{q}^{2}(\Delta-\delta / 3) \mathrm{mm} / \mathrm{s}^{2}$ for rectangular pulses.

Historically, the diffusion tensor [2] was the first model to describe the EAP by assuming it lies within the family of Gaussian distributions. However, this assumption limits its ability to describe complex tissue structures [3].

We can reconstruct the EAP without prior assumptions by using an inverse Fourier transform on dense acquisition schemes such as Diffusion Spectrum Imaging (DSI) [4]. However, DSI's dense sampling of q-space limits its clinical applicability. As a solution, analytical bases whose functions are eigenvectors of the Fourier transform have been proposed which provide compact representation of the 3D q-space signal. These bases capture the radial and angular properties of the diffusion signal by fitting a linear combination of orthogonal basis functions as $E(\mathbf{q})=\sum_{i}^{N} \mathbf{c}_{i} \Phi(\mathbf{q})$. They then describe the EAP as $P(\mathbf{r})=\mathbf{c}_{i} \Psi(\mathbf{r})$ where $\Psi(\mathbf{r})$ is the inverse Fourier transform of $\Phi(\mathbf{q})$. In particular, the recent Mean Apparent Propagator (MAP)-MRI basis [5], which is related to the preceding 3D Simple Harmonic Oscillator based Reconstruction and Estimation (3D-SHORE) basis [5], fits the signal extremely well by using anisotropic basis functions.

However, without proper regularization the basis fitting becomes highly unreliable in the presence of noise, making accurate EAP estimation impossible. As a solution we propose a fast and robust Laplacian regularization for the MAPMRI basis. We derive the Laplacian regularization functional analytically and from now on refer to our regularized MAPMRI as LMAP-MRI. We show that (1) MAP-MRI has more accurate microstructure recovery compared to classical techniques, (2) LMAP-MRI has lower signal fitting errors compared to regular MAP-MRI and a positivity constraint on the EAP [5], and (3) that LMAP-MRI has improved axon radius recovery on human data of the MGH Human Connectome Project [6].

\section{MATERIALS AND METHODS}

\subsection{Laplacian Regularization of MAP-MRI}

We propose to regularize MAP-MRI's basis fitting by minimizing the norm of its Laplacian. The MAP-MRI basis functions are given as a product of three 1D functions

$$
\begin{gathered}
\Phi_{N_{i}}(\mathbf{A}, \mathbf{q})=\phi_{n_{x(i)}}\left(u_{x}, q_{x}\right) \phi_{n_{y(i)}}\left(u_{y}, q_{y}\right) \phi_{n_{z(i)}}\left(u_{z}, q_{z}\right) \\
\text { with }\left\{\begin{array}{l}
\phi_{n}(u, q)=\frac{i^{-n}}{\sqrt{2^{n} n !}} e^{-2 \pi^{2} q^{2} u^{2}} H_{n}(2 \pi u q) \\
\mathbf{A}=\operatorname{Diag}\left(u_{x}^{2}, u_{y}^{2}, u_{z}^{2}\right)
\end{array}\right.
\end{gathered}
$$

with basis order $N_{i}=\left(n_{x(i)}, n_{y(i)}, n_{z(i)}\right)$. We find the diagonalized scaling factors $\mathbf{A}=\mathbf{R} \mathbf{A}^{\prime} \mathbf{R}^{T}$ by fitting a tensor $\mathbf{A}^{\prime}$, where $\mathbf{R}$ contains the tensor eigenvectors. We then rotate the data into the frame of reference using $\mathbf{R}$ and scale the basis functions using $\mathbf{A}$ along each direction. The anisotropic basis functions is what makes MAP-MRI quickly fit anisotropic 
data found in white matter tissue. MAP-MRI's zeroth order basis function is in fact a DTI tensor [2], while setting scaling factors $\left(u_{x}, u_{y}, u_{z}\right)$ to an isotropic $u_{0}$ makes the MAP-MRI basis equivalent to the 3D-SHORE basis [5].

We fit the MAP-MRI coefficients $\mathbf{c}$ to the data $\mathbf{y}=E(\mathbf{q})$ using regularized least-squares as $\mathbf{c}=\operatorname{argmin}_{\mathbf{c}}\|\mathbf{y}-\mathbf{Q c}\|^{2}+$ $\lambda R(\mathbf{c})$ where design matrix $\mathbf{Q} \in \mathbb{R}^{N_{\text {data }} \times N_{\text {coef }}}$ has elements $\mathbf{Q}_{i j}=\Phi_{N_{i}}\left(\mathbf{A}, \mathbf{q}_{j}\right)$, with $\mathbf{q}_{j}$ the q-space positions of the data. Parameter $\lambda$ weights the regularization functional $R(\mathbf{c})=$ $\int_{\mathbb{R}^{3}}\left\|\Delta E_{\mathbf{c}}(\mathbf{q})\right\|^{2} d \mathbf{q}$ with $\Delta E_{\mathbf{c}}(\mathbf{q})=\sum_{i} \mathbf{c}_{i} \Delta \Phi_{N_{i}}(\mathbf{A}, \mathbf{q})$ the Laplacian of the reconstructed signal. We then rewrite $R(\mathbf{c})=\mathbf{c}^{T} \mathbf{R} \mathbf{c}$ in quadratic form as

$$
\mathbf{R}_{i k}=\int_{\mathbb{R}^{3}} \Delta \Phi_{N_{i}}(\mathbf{q}, \mathbf{A}) \cdot \Delta \Phi_{N_{k}}(\mathbf{q}, \mathbf{A}) d \mathbf{q} .
$$

We solve this integral using the orthogonality of Hermite polynomials with respect to the weight function $e^{-x^{2}}$. This allows us to describe every entry in $\mathbf{R}_{i j}$ as a function of the anisotropic scaling factors $\left(u_{x}, u_{y}, u_{z}\right)$ and basis orders $\left(x_{i}, y_{i}, z_{i}\right)=\left(n_{x(i)}, n_{y(i)}, n_{z(i)}\right)$ as

$$
\begin{aligned}
\mathbf{R}_{i k} & =\frac{u_{x}^{3}}{u_{y} u_{z}} \mathrm{~S}_{x_{i}}^{x_{k}} \mathrm{U}_{y_{i}}^{y_{k}} \mathrm{U}_{z_{i}}^{z_{k}}+2 \frac{u_{x} u_{y}}{u_{z}} \mathrm{~T}_{x_{i}}^{x_{k}} \mathrm{~T}_{y_{i}}^{y_{k}} \mathrm{U}_{z_{i}}^{z_{k}} \\
& +\frac{u_{y}^{3}}{u_{z} u_{x}} \mathrm{~S}_{y_{i}}^{y_{k}} \mathrm{U}_{z_{i}}^{z_{k}} \mathrm{U}_{x_{i}}^{x_{k}}+2 \frac{u_{y} u_{z}}{u_{x}} \mathrm{~T}_{y_{i}}^{y_{k}} \mathrm{~T}_{z_{i}}^{z_{k}} \mathrm{U}_{x_{i}}^{x_{k}} \\
& +\frac{u_{z}^{3}}{u_{x} u_{y}} \mathrm{~S}_{z_{i}}^{z_{k}} \mathrm{U}_{x_{i}}^{x_{k}} \mathrm{U}_{y_{i}}^{y_{k}}+2 \frac{u_{x} u_{z}}{u_{y}} \mathrm{~T}_{x_{i}}^{x_{k}} \mathrm{~T}_{z_{i}}^{z_{k}} \mathrm{U}_{y_{i}}^{y_{k}}
\end{aligned}
$$

with functions $\mathrm{S}_{n}^{m}, \mathrm{~T}_{n}^{m}$ and $\mathrm{U}_{n}^{m}$ given as

$$
\begin{aligned}
& \mathrm{S}_{n}^{m}=2(-1)^{n} \pi^{7 / 2}\left(\delta_{n}^{m} 3\left(2 n^{2}+2 n+1\right)\right. \\
& +\delta_{n+2}^{m}(6+4 n) \sqrt{m ! / n !}+\delta_{n+4}^{m} \sqrt{m ! / n !} \\
& \left.\quad+\delta_{n}^{m+2}(6+4 m) \sqrt{n ! / m !}+\delta_{n}^{m+4} \sqrt{n ! / m !}\right) \\
& \mathrm{T}_{n}^{m}=(-1)^{n+1} \pi^{3 / 2}\left(\delta_{n}^{m}(1+2 n)\right. \\
& \left.\quad+\delta_{n}^{m+2} \sqrt{n(n-1)}+\delta_{n+2}^{m} \sqrt{m(m-1)}\right) \\
& \mathrm{U}_{n}^{m}=\delta_{n}^{m}(-1)^{n} /\left(2 \pi^{1 / 2}\right)
\end{aligned}
$$

with $\delta_{n}^{m}$ the Kronecker delta. We finally obtain the MAP-MRI coefficients through $\mathbf{c}=\left(\mathbf{Q}^{\top} \mathbf{Q}+\lambda \mathbf{R}\right)^{-1} \mathbf{Q}^{\top} \mathbf{y}$. We used generalized cross-validation [7] to find an optimal value of $\lambda=0.2$ in noisy data and set this value for all our experiments.

\subsection{Estimation of Axon Radii}

From the MAP-MRI coefficients $\mathbf{c}$ we can describe axon radii distributions using the Return-To-Axis Probability (RTAP) [5]. RTAP is defined directionally, representing the integrated probability that a spin diffuses along the axis of the axons, here defined as the main tensor eigenvector $\mathbf{R}_{\|}$.

The RTAP is only related to the axon radius if (1) axons are represented as parallel cylinders, (2) only the intra-axonal signal is considered and (3) the acquisition has short gradient pulses $(\delta \approx 0)$ and gradient separation time long enough for restricted diffusion $(\Delta \gg \delta)$. If these conditions are met we compute the axon radii as

$$
\langle R\rangle=\sqrt{1 /(R T A P \pi)} \text { with } R T A P=\int_{\mathbb{R}} P\left(\mathbf{R}_{\|} r\right) d r
$$

Deviation from these criteria will cause misestimation of the axon radius [8], which we quantify in Section 3.1.

\section{RESULTS}

We validate our MAP-MRI regularization on synthetic data using Camino [9] and on the MGH Human Connectome project (HCP) data set [6].

\subsection{Synthetic Validation and Microstructure Recovery}

We use the Camino diffusion MRI toolkit [9] to simulate the diffusion signal in voxels with parallel cylindrical axons with radii ranging from $0.3 \mu \mathrm{m}$ to $6 \mu \mathrm{m}$. We generate both data respecting all the conditions stated in Section $2.2(\delta \approx 0, \Delta \gg$ $\delta$ ) (I) and data using HCP settings $(\delta / \Delta=12.9 / 21.8 \mathrm{~ms})$ (II). We simulate according to the HCP parameters at b-values $\{0 ; 1,000 ; 3,000 ; 5,000 ; 10,000\} \mathrm{mm} / \mathrm{s}^{2}$ with $\{40 ; 64 ; 64 ;$ $128 ; 256\}$ gradient directions, and sample (I) such that we sample the same q-space positions of (II) by inversely scaling the diffusion gradient strength with $\delta$.

We first consider the noiseless case of the optimal data and compute the axon radii with MAP-MRI, SHORE and DTI (Figure 1a). It is clear that MAP-MRI is able to distinguish smaller axon radii than the other techniques thanks to its anisotropic basis functions. We then take one axon radius of $1.8 \mu \mathrm{m}$ and apply noise 300 different times at a realistic $S N R=15$. We compute the axon radius with increasing radial order using either the positivity constraint, our Laplacian or both. We find that both methods come close to the ground truth but our Laplacian, in blue, has significantly lower standard deviations at higher radial orders (Figures $1 \mathrm{~b}$ and 1c). We then compare the different methods based on the Mean Squared Error (MSE) of the noisy signal reconstruction compared to the ground truth. We used radial order 4 and truncated the outer two shells such that bmax $=3000 \mathrm{~mm} / \mathrm{s}^{2}$. We show this experiment in Figures 1d and 1e: our Laplacian regularization, again in blue, has lower MSE values and standard deviations. Finally, we observe the effect of truncating the outer shells on the noiseless HCP simulated data on axon radius estimation (Figure 1f). In the full data we find that the suboptimal HCP parameters lead to underestimated axon radii until a plateau is reached at around $1.3 \mu \mathrm{m}$. Truncation of the outer shells leads to higher axon radii estimates. 


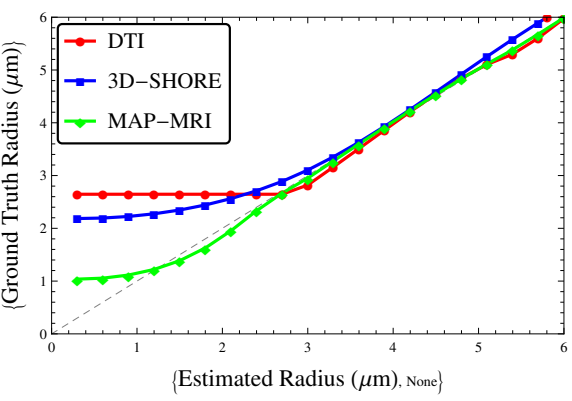

(a) Axon Radius Estimation Optimal Settings

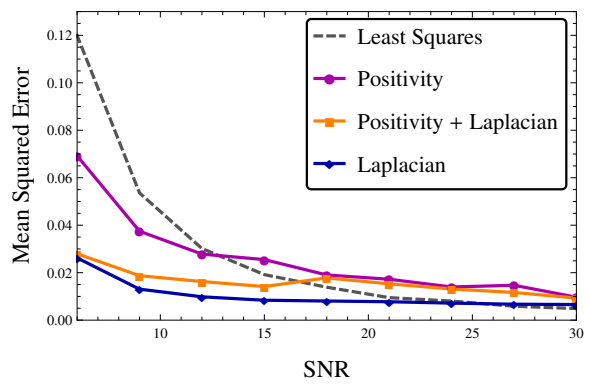

(d) MAP-MRI signal fitting bmax $=3,000$

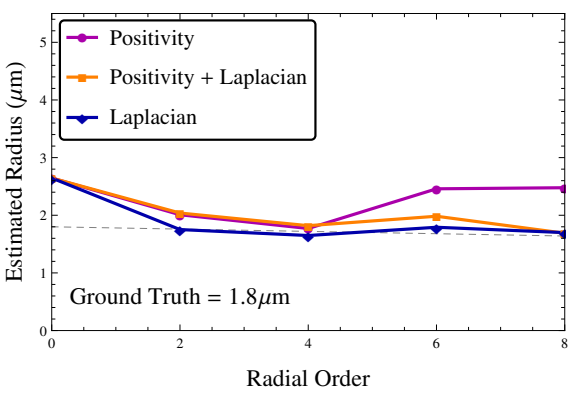

(b) MAP-MRI Convergence bmax $=10,000$

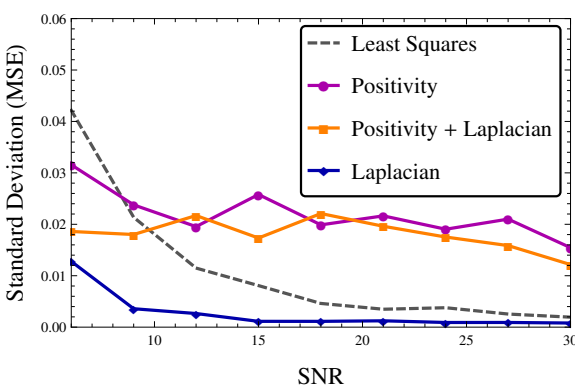

(e) MAP-MRI standard deviation bmax $=3,000$

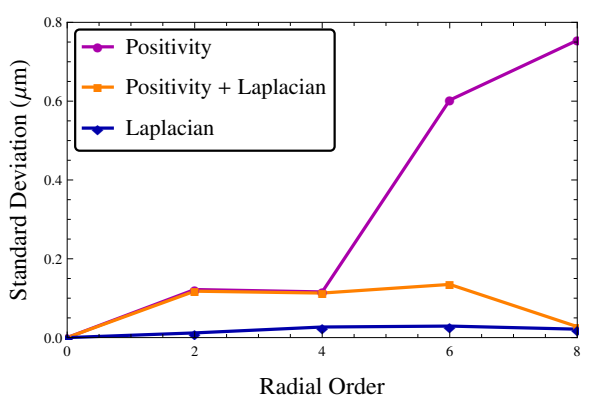

(c) MAP-MRI standard deviation bmax $=10,000$

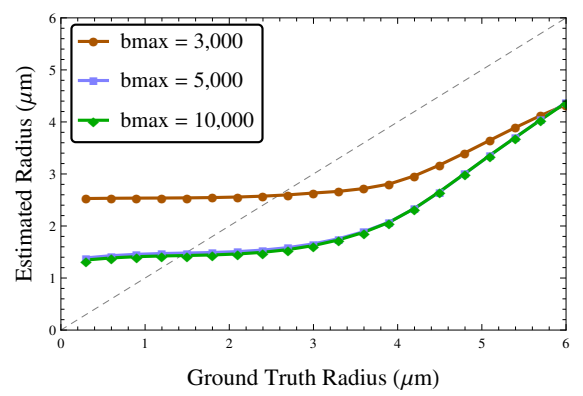

(f) MAP-MRI effect of truncation HCP settings

Fig. 1: Results on synthetic Camino experiments. (a) shows a comparison of axon estimations between MAP-MRI, SHORE and DTI on optimal data. (b) compares the average MAP-MRI axon radius estimate for different radial orders and methods with known ground truth. (c) shows the standard deviation. (d) quantifies the average MAP-MRI signal reconstruction for different SNR levels based on MSE. (e) gives the standard deviations. (f) shows the effect truncating the outer sampling shells on the axon radius estimation in HCP simulated data.

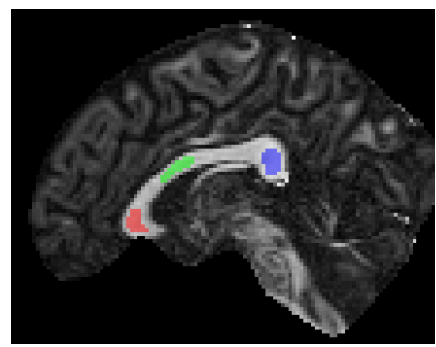

Fig. 2: Segmentation of the splenium, midbody and genu (red, green and blue) of the corpus callosum.

\subsection{Microstructure on the Human Connectome Project}

In our second experiment we estimate axon radii in the corpus callosum data in a subject of the MGH Human Connectome Project data [6]. In this area the white matter is known to be highly coherent, and that axon radii are larger in the midbody than in the genu and splenium [10]. We segment these areas (Figure 2) and compute the axon radii in each of these areas using our Laplacian or the positivity constraint (Figure 3 ). For both methods we find larger axon radii in the midbody (green) than in the genu in splenium (red and blue), though we always overestimate compared to the real radius of around $0.1 \mu m-1 \mu m$ [10]. When we truncate the outer shells we find higher axon radius estimates, but both methods remain able to distinguish between axon populations.

\section{DISCUSSION AND CONCLUSION}

In this paper we proposed a new regularization method for the recent MAP-MRI basis. We derived the Laplacian functional analytically, and showed on synthetic Camino data that a Laplacian-regularized MAP-MRI basis (LMAP-MRI) has more robust and accurate signal reconstruction and axon radius recovery than using a positivity constraint on the EAP. We find that this effect is more distinct when dealing with noisy, more undersampled acquisition data. Even combining the two methods does not decrease the reconstruction error further. Our regularization method is also fast, having the same speed as ordinary least squares fitting. We also show that MAP-MRI is able to retrieve axon radii on synthetic data until significantly smaller axon radii compared to 3D-SHORE and DTI. We finally validate our method on human data from the HCP project. We find that LMAP-MRI can actually distinguish axon populations in the corpus callosum, even in truncated data. However, overall we see axon radii are overestimated.

In closing, we find in both our synthetic and human experiments that MAP-MRI is superior to classical techniques 

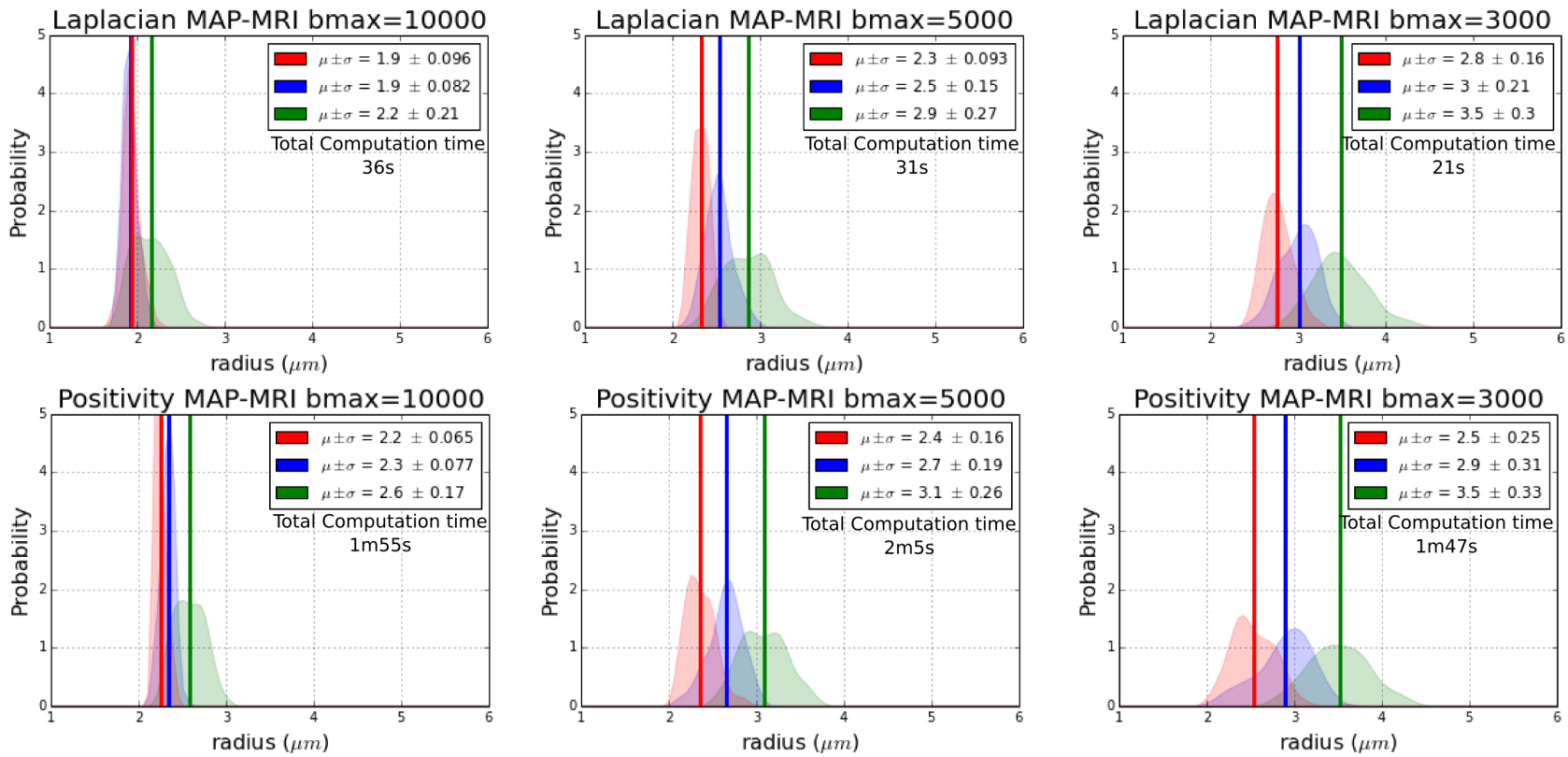

Fig. 3: Axon radius estimation in the corpus callosum using either Laplacian regularization or unregularized with a positivity constraint. We correctly find larger axon radii in the midbody (green) than in the genu and splenium (blue and red). The laplacian generally estimates populations with smaller standard deviations and finds slightly smaller radii in the $b=10,000$ data but larger for $b=3,000$ data. In terms of computation time our Laplacian approach is 4 times faster than the positivity contraint.

in terms of axon radius recovery, and our LMAP-MRI provides both faster and more robust signal fitting and axon radius recovery compared to both regular MAP-MRI and using a positivity constraint when facing noisy, undersampled data.

\section{Acknowledgments}

Data used in the preparation of this work were obtained from the Human Connectome Project (HCP) database

(https://ida.loni.usc.edu/login.jsp). The HCP project is supported by the National Institute of Dental and Craniofacial Research (NIDCR), the National Institute of Mental Health (NIMH) and the National Institute of Neurological Disorders and Stroke (NINDS). This work was partly supported by the French ANR "MOSIFAH" under ANR-13-MONU-0009-01.

\section{REFERENCES}

[1] Stejskal. "Use of Spin Echoes in a Pulsed Magnetic-Field Gradient Study Anisotropic Restricted Diffusion Flow." J CHEM PHYS 43.10, pp. 3597-3603, 1965.

[2] Basser et al. "Estimation of the Effective Self-Diffusion Tensor from the NMR Spin Echo." J MAGN RESON, Series B 103.3, pp. 247-254, 1994.

[3] Assaf Yaniv and Ofer Pasternak. "Diffusion tensor imag- ing (DTI)-based white matter mapping in brain research: a review." J Mol NEUROSCI 34.1, pp. 51-61, 2008.

[4] Wedeen et al. "Mapping Complex Tissue Architecture with Diffusion Spectrum Magnetic Resonance Imaging." MAGNET RESON MED 54.6, pp. 1377-1386, 2005.

[5] Özarslan et al. "Mean Apparent Propagator (MAP) MRI: A novel Diffusion Imaging Method for Mapping Tissue Microstructure.” NeuroImage 78, pp. 16-32, 2013.

[6] Setsompop et al. "Pushing the limits of in vivo diffusion MRI for the Human Connectome Project." NeuroImage 80, pp. 220-233, 2013.

[7] Craven et al. "Smoothing Noisy Data with Spline Functions." NUMER MATH 31.4, pp. 377-403, 1978.

[8] Bar-Shir et al. "The effect of the diffusion time and pulse gradient duration ratio on the diffraction pattern and the structural information estimated from q-space diffusion MR: Experiments and simulations." J MAGN RESON 194.2, pp. 230-236, 2008.

[9] Cook et al. "Camino: Open-Source Diffusion-MRI Reconstruction and Processing", 14th Scientific Meeting of the International Society for Magnetic Resonance in Medicine, vol. 2759, May 2006.

[10] Aboitiz et al. "Fiber composition of the human corpus callosum.” Brain research 598.1, pp. 143-153, 1992. 